\title{
Panicle Apical Abortion 3 Controls Panicle Development and Seed Size in Rice
}

\author{
Fayu Yang ${ }^{\dagger} \mathbb{D}$, Mao Xiong ${ }^{\dagger}$, Mingjiang Huang ${ }^{\dagger}$, Zhongcheng Li, Ziyi Wang, Honghui Zhu, Rui Chen, Lu Lu, \\ Qinglan Cheng, Yan Wang, Jun Tang, Hui Zhuang and Yunfeng Li ${ }^{*}$
}

\begin{abstract}
Background: In rice, panicle apical abortion is a common phenomenon that usually results in a decreased number of branches and grains per panicle, and consequently a reduced grain yield. A better understanding of the molecular mechanism of panicle abortion is thus critical for maintaining and increasing rice production.

Results: We reported a new rice mutant panicle apical abortion 3 (paa3), which exhibited severe abortion of spikelet development on the upper part of the branches as well as decreased grain size over the whole panicle. Using mapping-based clone, the PAA3 was characterized as the LOC_Os04956160 gene, encoding an $\mathrm{H}^{+}$-ATPase. The PAA3 was expressed highly in the stem and panicle, and its protein was localized in the plasma membrane. Our data further showed that PAA3 played an important role in maintaining normal panicle development by participating in the removal of reactive oxygen species (ROS) in rice.

Conclusions: Our studies suggested that PAA3 might function to remove ROS, the accumulation of which leads to programmed cell death, and ultimately panicle apical abortion and decreased seed size in the paa3 panicle.
\end{abstract}

Keywords: Rice, Panicle apical abortion, Programmed cell death, $\mathrm{H}^{+}$-ATPase

\section{Background}

Rice (Oryza sativa) is one of the most important food crops in the world and the major staple food for more than half the world's population (Takeda and Matsuoka, 2008). Rice yield is determined by three main component traits: number of panicles, number of grains per panicle, and grain weight (Xing and Zhang, 2010). Therefore, the panicle plays a key role in contributing directly to yield, and the achievement of optimal panicle structure, size, and shape is one of the goals in highyield breeding (Sakamoto and Matsuoka, 2004).

In the development of the inflorescence, the transition from shoot meristems to axillary meristems (AMs) determines the complexity of the inflorescence architecture

\footnotetext{
* Correspondence: liyf1980@swu.edu.cn

${ }^{\dagger}$ Fayu Yang, Mao Xiong and Mingjiang Huang contributed equally to this work.

Rice Research Institute, Key Laboratory of Application and Safety Control of Genetically Modified Crops, Academy of Agricultural Sciences, Southwest University, Chongqing 400715, China
}

\section{Springer Open}

(Huijser and Schmid, 2011). The rice panicle has a fourorder inflorescence structure, comprising the main axis, primary branches (PBs), secondary branches (SBs), lateral spikelet (LS), and terminal spikelet (TS), and all of which are originated from the AMs. The past few years have seen the identification of a number of regulatory genes involved in the determination of panicle architecture, including lymphocyte transmembrane adaptor 1 (LAX1), LAX2, and MONOCULM 1. These genes participate in the initiation, formation, and maintenance of AMs in the rice panicle and are regulators of panicle architecture. They all encode transcription factor and the loss-of-function mutants display greatly reduced numbers of branches and spikelets (Komatsu et al., 2003; Li et al., 2003; Tabuchi et al., 2011). The transition from branch meristem to spikelet meristem is also considered a key process related to panicle architecture. RETICULOCALBIN 1 (RCN1) and RCN2 maintain the branch meristem and control its fate in rice, and their overexpression leads to the delayed transition from

(c) The Author(s). 2021 Open Access This article is licensed under a Creative Commons Attribution 4.0 International License, which permits use, sharing, adaptation, distribution and reproduction in any medium or format, as long as you give appropriate credit to the original author(s) and the source, provide a link to the Creative Commons licence, and indicate if changes were made. The images or other third party material in this article are included in the article's Creative Commons licence, unless indicated otherwise in a credit line to the material. If material is not included in the article's Creative Commons licence and your intended use is not permitted by statutory regulation or exceeds the permitted use, you will need to obtain permission directly from the copyright holder. To view a copy of this licence, visit http://creativecommons.org/licenses/by/4.0/. 
branch meristem to spikelet meristem, and then to more branches and spikelets (Nakagawa et al., 2002). ABBERANT PANICLE ORGANIZATION 2 (APO2)/RFL can be activated by the DNA-binding one zinc finger DOFdomain transcription activator SHORT PANICLE 3 (SP3), and interacts with APO1 to delay the transition from branch meristem to spikelet meristem. In the mutants apo1, apo 2 and sp3, the numbers of branches and spikelets are all significantly reduced (Rao et al., 2008; Ikeda-Kawakatsu et al., 2012). In addition, TAW1 encodes a nuclear protein that participates in determining the fate of the branch meristem. In the gain-of-function mutant taw1-d, the branch meristem activity is increased and the differentiation of the spikelet meristem is delayed, which promotes the formation of more SBs and spikelets (Yoshida et al., 2013). Plant hormones such as cytokinin also play crucial roles in panicle architecture. Grain number 1a (Gn1a), a major quantitative trait locus for grain number per panicle, encodes cytokinin oxidase which is responsible for the degradation of cytokinin. In the natural allelic variant with low expression of $G n 1 a$, the accumulation of cytokinin leads to greatly increase branch number, spikelet number, and grain yield (Ashikari et al., 2005). Multiple genes such as DROUGHT AND SALT TOLERANCE (DST), VIN3-LIKE 2 (VIL2) and LARGER PANICLE (LP) (Li et al., 2011; Li et al., 2013; Yang et al., 2019) participate in panicle development by regulating the expression of Gnla. The microRNA156- IDEAL PLANT ARCHITECTURE 1DENSE AND ERECT PANICLE 1 (MicroRNA156IPA1-DEP1) pathway probably regulates panicle development through cytokinin, in which IPA1 can directly activate DEP1 expression by interacting with its promoter, and then the high expression of DEP1 represses Gn1a (Huang et al., 2009; Lu et al., 2013).

After the panicle architecture is established, normal spikelet growth is very important for the final yield. Panicle abortion occurs at either the top or basal parts of the panicle. Spikelet growth often stops at the apex of the panicle and/or branches under disadvantageous conditions (malnutrition, extreme temperatures, shading, and water stress) and genetic alteration, which is usually termed "panicle apical abortion" (Kobayasi et al., 2001; Kato et al., 2008; Bai et al., 2015). To date, only a few studies have set out to characterize the molecular mechanism of panicle abortion. The TOTOU1 gene was the first cloned pleiotropy gene associated with panicle degeneration, through its encoding of cyclic adenosine monophosphate receptor protein inhibitors. TOTOU1 mutation leads to panicle apical abortion and other defects including tiller reduction, leaf tip degeneration, and dwarfing (Bai et al., 2015). SQUAMOSA PROMOTERBINDING PROTEIN-LIKE 6 (SPL6) functions as a transcriptional repressor of INOSITOL-REQUIRING
ENZYME (IRE1), and acts as an essential survival factor for the suppression of persistent or intense stress in the endoplasmic reticulum, leading ultimately to cell death in rice. The spl6-1 mutant displays hyperactivation of $I R E 1$, leading to cell death in spikelets in the panicle apex (Wang et al., 2018a, b). ALUMINUM-ACTIVATE D MALATE TRANSPORTER 7 (OsALMT7) is a malate transporter that functions in the development of panicle apical portions, and its mutation also results in reduced malate and cell death, particularly at the apical portion of the panicle (Heng et al., 2018). The paa1019 mutant is specifically defective in panicle development. PAA1019 encodes OsCIPK31, a calcineurin B-like-interacting protein kinase that affects the development of panicle apical spikelets (Peng et al., 2018). The degenerated panicle and partial sterility 1 (dps1) mutant also show panicle apical degeneration and reduced fertility in middle spikelets. In addition, the amounts of cuticular wax and cuticle are reduced significantly in $d p s 1$ anthers, and the accumulation of reactive oxygen species (ROS), lower antioxidant activity, and increased programmed cell death (PCD) have all been observed (Zafar et al., 2019).

In this study, we report a novel rice mutant panicle apical abortion 3 (paa3), which exhibits the degeneration of spikelets at the tops of panicles during the late stage of panicle development. The results of gene cloning and complementation tests indicate that $P A A 3$ is LOC_Os04g56160, encoding an $\mathrm{H}^{+}$-ATPase. Our data further suggest that $P A A 3$ might function to remove peroxides, and the accumulation of ROS leads to PCD and ultimately panicle apical abortion in the paa3 mutant.

\section{Results}

The paa3 Mutant Displayed a Semi-Dwarf Phenotype and Severe Panicle Apical Abortion

The paa3 mutant exhibited decreased plant height and grain defects (Fig. 1). The dwarf phenotype in the paa3 mutant was first observed at the tillering stage (Fig. 1a), and the semi-dwarf phenotype lasted until maturity stage (Fig. 1b). Detailed analyses show that the lengths of both the internodes (from 1 to 4 ) and the panicle in the paa3 mutant were all significantly reduced compared to the wild type (WT), and internode 5 was not elongated in the paa3 mutant but was elongated in the WT (Fig. 1c, i). Both the grain number and size in the paa3 mutant were also affected (Fig. 1e-h). The numbers of PBs and SBs were all sharply reduced compared with the WT (Fig. 11, m), which resulted in a significant reduction in the spikelet number per panicle (including the aborted spikelet in paa3 mutant) (Fig. 1n). The number of grains in the paa3 panicles was also significantly decreased compared to the WT (Fig. 1o). In addition, both grain length and grain width, as well as 1000-grain weight in 


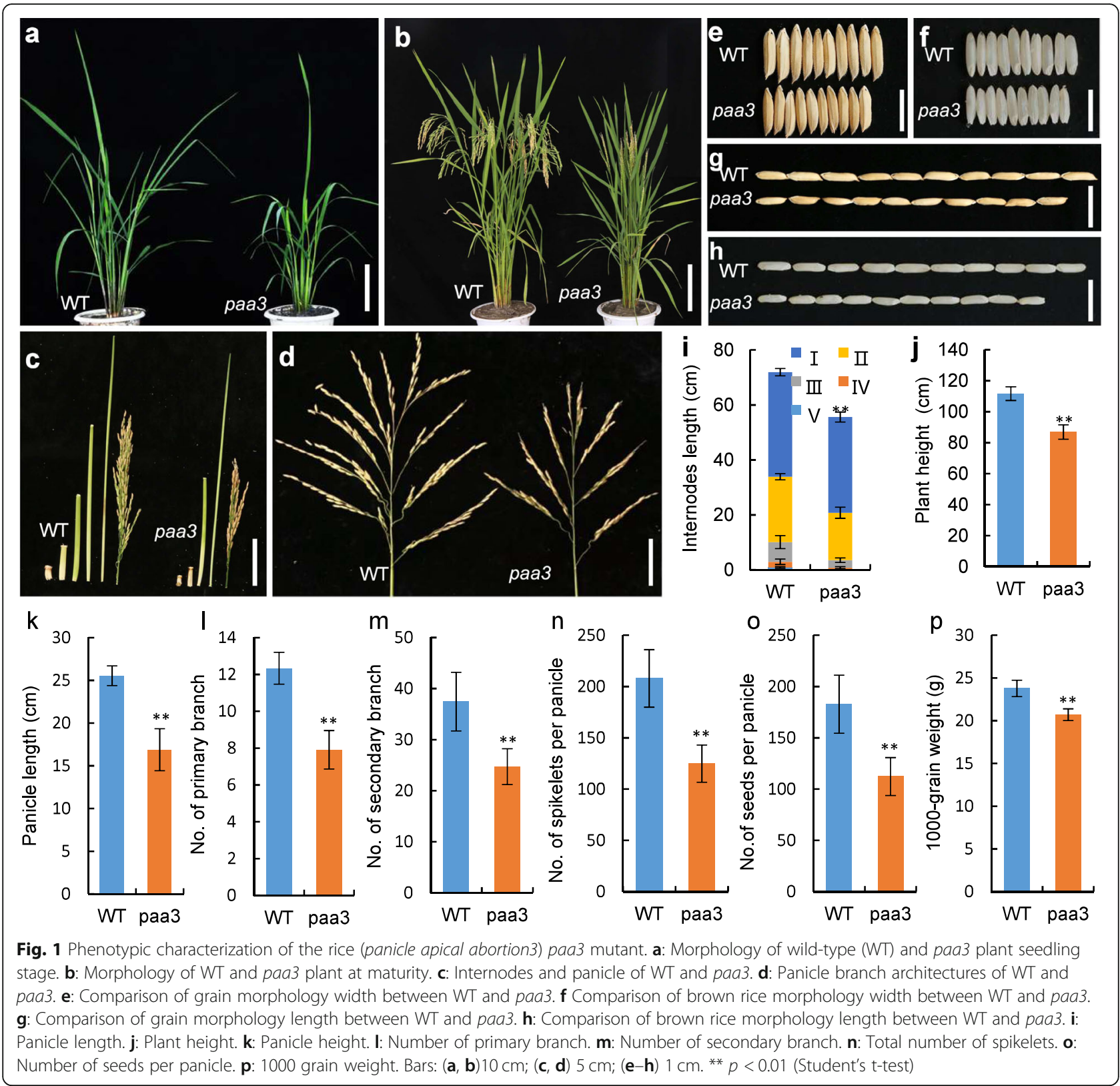

the paa3 mutant were all significantly reduced compared to those in the WT (Fig. 1e-h, p). In particular, unlike the WT plants in which all the spikelets developed normally, obvious panicle apical abortion was found in the paa3 panicle, in which the spikelets in the apical part of the panicle showed termination of development and were unable to seed (Fig. 1d). To determine when the abortion occurred, we investigated a series of mutant panicles at nine stages according to panicle length $(\sim 1$, $\sim 3, \sim 5, \sim 7, \sim 9, \sim 11, \sim 13, \sim 15$, and $\sim 17 \mathrm{~cm})$. The paa3 spikelets at the top of the panicles began to show developmental delay at $11 \mathrm{~cm}$, and the abortion phenotype became increasingly obvious from $\sim 13$ to $\sim 17 \mathrm{~cm}$ (Fig. 2a1-a9). We further observed in detail the spikelets at the top of the paa3 panicles from $\sim 9$ to $\sim 17 \mathrm{~cm}$ (Fig. 2b1-b10). At the $\sim 9 \mathrm{~cm}$ panicles, there were no obvious differences between the WT and the paa3 spikelets (Fig. 2b1, b2). However, some of the spikelets at the top of the $\sim 11$ and $\sim 13 \mathrm{~cm}$ panicles displayed white hulls and smaller size in paa3 mutants, whereas the WT had green hulls and were larger in size (Fig. 2b3-b6). In about $\sim 15 \mathrm{~cm}$ paa3 panicles, the spikelet organs at the top of the panicles began to browning (Fig. 2b7, b8), and in the $\sim 17 \mathrm{~cm}$ paa3 panicles, the spikelets were completely dry (Fig. 2b9, b10). According to these results, we speculated that the spikelets located at the top of the paa3 panicles stopped growing at $\sim 9$ to $11 \mathrm{~cm}$, after which cell death occurred gradually. We also observed 


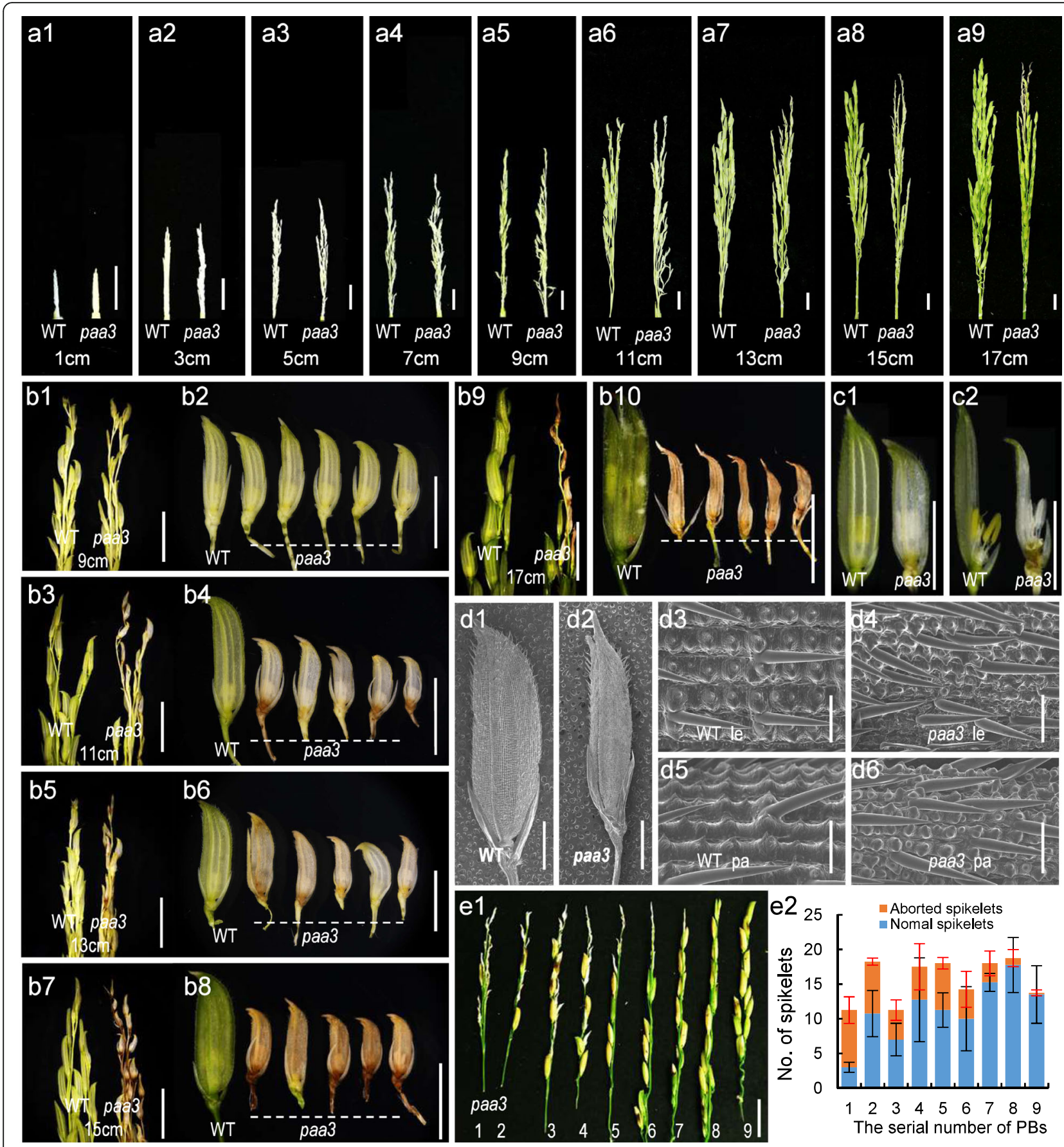

Fig. 2 Characteristics of abortion spikelets. a: Representative images of WT (left) and paa3 (right) developing panicles, showing different stages as indicated by panicle length:1 cm (a) $, 3 \mathrm{~cm}(\mathbf{a} 2), 5 \mathrm{~cm}(\mathbf{a} 3), 7 \mathrm{~cm}(\mathbf{a} 4), 9 \mathrm{~cm}(\mathbf{a} 5), 11 \mathrm{~cm}(\mathbf{a} 6), 13 \mathrm{~cm}(\mathbf{a} 7), 15 \mathrm{~cm}(\mathbf{a} 8)$ and $17 \mathrm{~cm}(\mathbf{a} 9)$. b: The apical developing panicles, showing different stages as indicated by panicle length: $9 \mathrm{~cm}(\mathbf{b} 1, \mathbf{b} 2), 11 \mathrm{~cm}(\mathbf{b} 3, \mathbf{b} 4), 13 \mathrm{~cm}(\mathbf{b} 5, \mathbf{b} 6), 15 \mathrm{~cm}(\mathbf{b} 7, \mathbf{b} 8), 17 \mathrm{~cm}$ (b9, b10). c: Top spikelet in paa3 mutants and WT; Total spikelet(c1), Spikelet without lemma(c2). (panicle length =11 cm): $\mathbf{d}$ : Scanning electron microscopy observation of top spikelet in paa3 mutant and WT: total spikelet of WT(d1), total spikelet of paa3(d2), WT lemma (d3), paa3 lemma (d4), WT palea (d5). paa3 palea (d6). e: Analysis of the aborted panicle in paa3: The image of the primary branch of paa3 panicle; Arabic numerals (1-9) denote the serial number of PBs(e1), Analysis of degenerated spikelets in each primary branch of paa3 in e1(e2). Bars: (a) 1-a99, e1)1 cm; (b1b10 and c1-c2) $5 \mathrm{~mm} ;(\mathbf{d} 1-\mathbf{d} 2) 2 \mathrm{~mm} ;(\mathbf{d} 3-\mathbf{d} 4) 120 \mu \mathrm{m}$

apical spikelets with $\sim 9 \mathrm{~cm}$ panicles in WT and paa3 mutant. And found that the stamen exhibited a hydrated phenotype (Fig. 2c1-c2). We further used scanning electron microscopy to observe the hull development in the $\sim 11 \mathrm{~cm}$ panicles and found the size of the epidemic cells in both the lemma and palea of paa3 were smaller 
than those in the WT (Fig. 2d1-d6). We conducted statistics on the abortion rate of panicles of wild type and mutants, and found that the abortion rate of mutants could reach $18.25 \%$ (Table S1). In addition, we observed the degree of spikelet abortion among the PBs for paa3 by statistical analysis of 15 paa3 panicles from 15 individual plants at the mature stage. The degree of spikelet abortion increased gradually from the lower to the upper PBs, and the upper spikelets were degenerated at a higher rate than the lower ones (Fig. 2e1, e2).

\section{Map-Based Cloning of the PAA3 Gene}

The paa3 mutant was crossed with the sterile line $56 \mathrm{~s}$ to obtain the F1 generation, and the F1 generation was self-crossed to obtain the F2 population. All F1 plants exhibited a normal phenotype, while trait segregation occurred in the F2 population. The number of plants in the F2 population was 196, of which 138 were normal, 58 displayed paa3 mutational phenotype, for a segregate ratio of 3.379:1, which was in accordance with the standard 3:1 of the chi-square test. Indicating that the mutant trait was controlled by a single recessive gene. In the $F_{2}$ progeny, 58 individuals exhibiting a mutant phenotype were used as the mapping population. Using bulked segregant analysis, the $P A A 3$ gene was mapped to the long arm of chromosome 4 within an approximately $88 \mathrm{~Kb}$ region between the simple-sequence repeat (SSR) markers CHR4-P118-6 and CHR4-P118-9. In this region, a single-nucleotide substitution from $\mathrm{G}$ to A (Gly to Asp) was identified in the 13th exon of LOC_ Os04g56160 (Fig. 3a). The complementary expression vector containing the LOC_OsO4g56160 coding sequence (6406 bp), the $3251 \mathrm{bp}$ upstream sequence from the start codon, and the $1068 \mathrm{bp}$ downstream sequence from the stop codon was then transformed into the paa3 mutants. In total, 28 transgenic plants were obtained, of which 15 showed rescued of the mutated phenotypes (Fig. 3b, c). We used two pairs of primers for amplification to detect the 15 transformants (comF1-GUSR1 for exogenous vector; comF2-comR2 for endogenic sites) (Fig. S1a). The sequencing results showed that the comF1-GUSR1 fragment was homozygous WT genotype and the comF2comR2 fragment was homozygous mutation genotype, indicating that the exogenous complementary plasmid
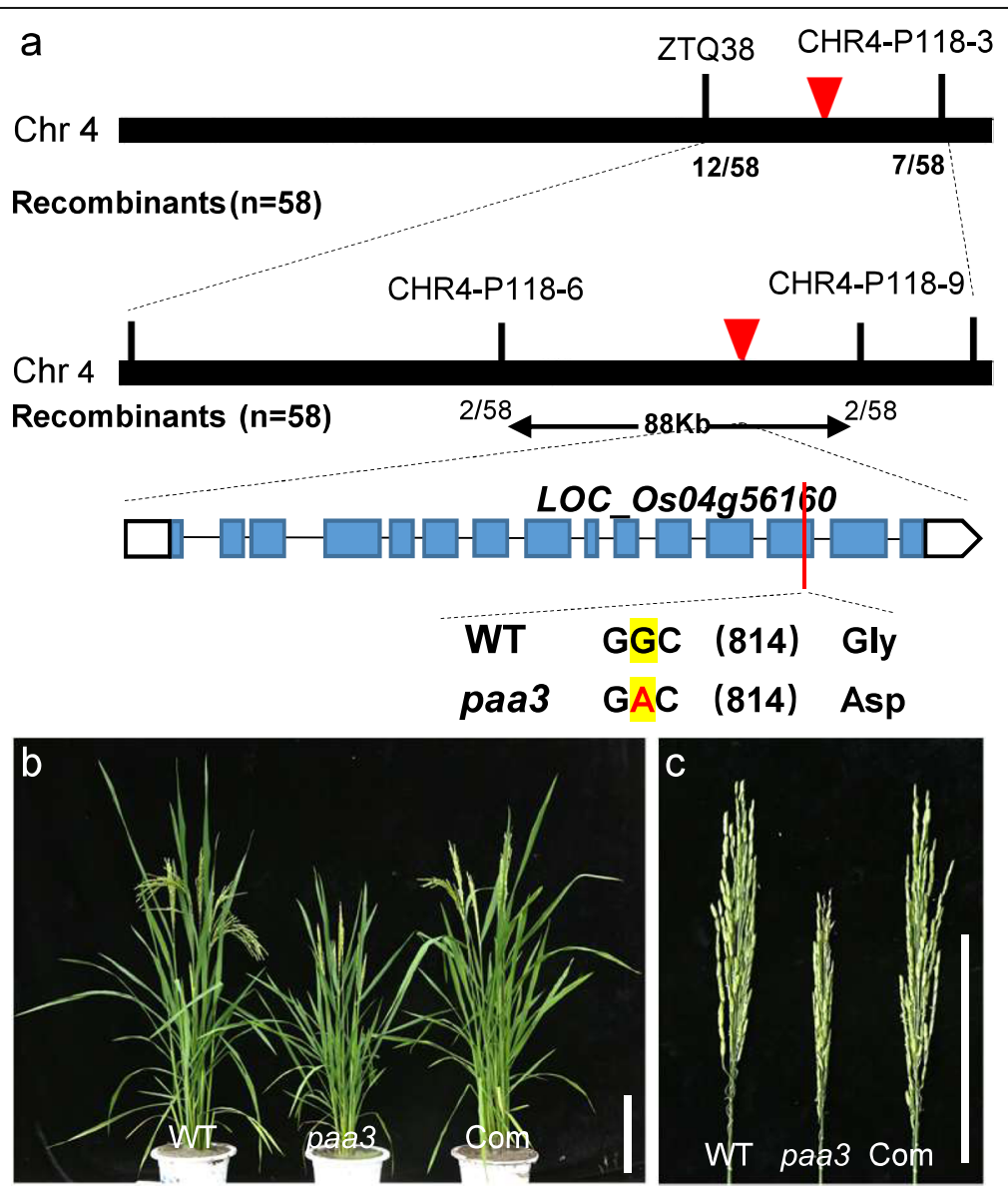

Fig. 3 Molecular identification of PAA3 and genetic complementation test. a: Fine mapping of the PAA3 gene. $\mathbf{b}$, c: Genetic complementation of paa3. b: Image of whole plants. c: Image of panicles. Bars (b, c) $15 \mathrm{~cm}$ 
had been transformed successfully with the paa3 mutant (Fig. S1b). Therefore, these results together indicated that $L O C \_O s 04 g 56160$ was the PAA3 gene.

\section{Expression Pattern Analysis and Subcellular Location of the $P A A 3$}

To explore the spatiotemporal expression of $P A A 3$ in rice, we firstly applied quantitative PCR (qPCR) to examine PAA3 expression in the WT plants. Expression of $P A A 3$ was detected in all rice organs analyzed, with relatively higher expression in the stems, roots, and panicles and lower expression in leaves and sheath (Fig. 4a). Next, we generated stable transgenic rice plants expressing the $\beta$-glucuronidase (GUS) reporter gene driven by a $3138 \mathrm{bp}$ promoter sequence of $P A A 3$ (Fig. 4b). Strong GUS staining was detected in the roots, stems, young panicles, and spikelets (Fig. $4 \mathrm{c}-\mathrm{e}, \mathrm{h}$ ), while faint staining was observed in the leaves and sheathes (Fig. 5f, g), similar to the qPCR results. Notably, a strong GUS signal was detected in the hulls of the spikelets and stems (Fig. $4 \mathrm{i}-\mathrm{j})$.

In this study, to clarify the subcellular location of PAA3, we fused it with the green fluorescent protein (GFP) reporter gene and expressed the PAA3:GFP fusion protein in rice protoplasts. In cells expressing $35 \mathrm{~s}:$ : PAA3:GFP protein, the GFP signal was observed mainly in the plasma membrane, and it was indeed colocalized with MF4-64 on the plasma membrane in the protoplasts (Fig. 4k). In the control group, $35 \mathrm{~s}$ promoterdriven GFP signals could be seen in the whole protoplast (Fig. 4k). In Nicotiana benthamiana leaves, the GFP fluorescence of $35 \mathrm{~s}:$ PAA3:GFP was also localized to the plasma membrane as labelled by MF4-64 (Fig. S2). These results suggested that PAA3 protein was localized to the plasma membrane.

In order to explore whether the change of single nucleotide affected the subcellular location of mutated PAA3 protein, we also fused it with the GFP reporter gene and expressed the paa3:GFP fusion protein in rice protoplasts (35 s::paa3:GFP Fig. 4k). The GFP signal of 35 s::paa3:GFP was located in the whole protoplasts highly consistent with the signal of 35 s::GFP. These results suggest that mutated paa 3 protein lost the location of plasma membrane because of its the single nucleotide substitution from $\mathrm{G}$ to $\mathrm{A}$.

\section{PCD Occurs in the Spikelets of paa3 Panicles}

As mentioned earlier, we found that the paa3 spikelets at the top of the panicles began to show developmental delay at $11 \mathrm{~cm}$ (Fig. 2). Trypan blue staining was used to detect the level of cell death at $\sim 13 \mathrm{~cm}$ in the WT and paa3 spikelets at the top of the panicles, and significantly deeper staining was observed in the paa3 spikelets than in the WT (Fig. S3). Therefore, these results indicated that the panicle apical abortion related to cell death in spikelet development during late paa3 panicles development. To examine further if the panicle abortion phenotype was related to the PCD, we performed a terminal deoxynucleotidyl transferase-mediated dUTP nick-end labelling (TUNEL) assay, which showed nuclear DNA fragmentation at the single-cell level. In the $\sim 7 \mathrm{~cm}$ panicles, no obvious TUNEL signal was detected in either the WT or the paa3 spikelet (Fig. 5a1-f1, a2f2). In the $\sim 11 \mathrm{~cm}$ panicles, however, strong TUNEL signals were observed in the anthers of the paa3 spikelets, and parts of the hull cells in the paa3 spikelets also showed clear TUNEL signals (Fig. 5j1-11, j2-12), whereas no TUNEL signal was seen in the WT spikelets (Fig. 5 g1-i1, i2-i2). In the $\sim 15 \mathrm{~cm}$ panicles, there was still no TUNEL signal in the WT spikelets (Fig. $5 \mathrm{~m} 1-\mathrm{o} 1, \mathrm{~m} 2-$ o2), but the whole of the spikelet (including hulls, stamens, gynoecium and sterile lemma) in the paa3 mutant showed very strong TUNEL signals (Fig. 5p1-r1, p2-r2). These results suggest that DNA fragmentation and cell death started to occur in the paa3 spikelets between the stages of $\sim 7-\sim 11 \mathrm{~cm}$, and reached a limitation at the stage of $\sim 15 \mathrm{~cm}$, consistent with the results of phenotypic analysis.

\section{Overaccumulation of ROS Induces PCD in Panicle Apical Spikelets}

ROS act as an important trigger of PCD, and excessive accumulation of hydrogen peroxide $\left(\mathrm{H}_{2} \mathrm{O}_{2}\right)$ can trigger cell death (Mittler, 2017). We used DAB staining to qualitatively detect the content of $\mathrm{H}_{2} \mathrm{O}_{2}$. In the paa3 panicles, DAB staining had revealed higher levels of ROS accumulation than that in the WT (Fig. S4). Here, we further measured $\mathrm{H}_{2} \mathrm{O}_{2}$ content in panicles of WT and paa3, and found an $\mathrm{H}_{2} \mathrm{O}_{2}$ blast at the $\sim 11 \mathrm{~cm}$ stage in the paa3 panicle (Fig. 6a). Malondialdehyde (MDA) accumulation is considered to be an indicator of lipid peroxidation and cell death (Chen and Murata, 2002). We therefore measured MDA content and found that the MDA levels were raised significantly in the 11 and 15 cm paa3 panicles compared to the WT (Fig. 6b).

Next, some of the genes related to ROS or PCD were investigated. Vacuolar processing enzymes (VPEs) are involved in PCD in Arabidopsis (Kuroyanagi et al., 2005). There are only four VPE homologs (OsVPE1, OsVPE2, OsVPE3, OsVPE4) in rice, of which OsVPE2 and OsVPE3 play crucial roles in $\mathrm{H}_{2} \mathrm{O}_{2}$-induced PCD (Deng et al., 2011). We therefore measured the expression of both OsVPE2 and OsVPE3 in the WT and the paa3 panicle at the $\sim 7, \sim 11$, and $15 \mathrm{~cm}$ stages. The expression of OsVPE2 in paa3 was similar to that of the WT at the $\sim 7 \mathrm{~cm}$ stage, but was increased significantly at the $\sim 11$ and $\sim 15 \mathrm{~cm}$ stages compared to those of the WT (Fig. 6c). The 


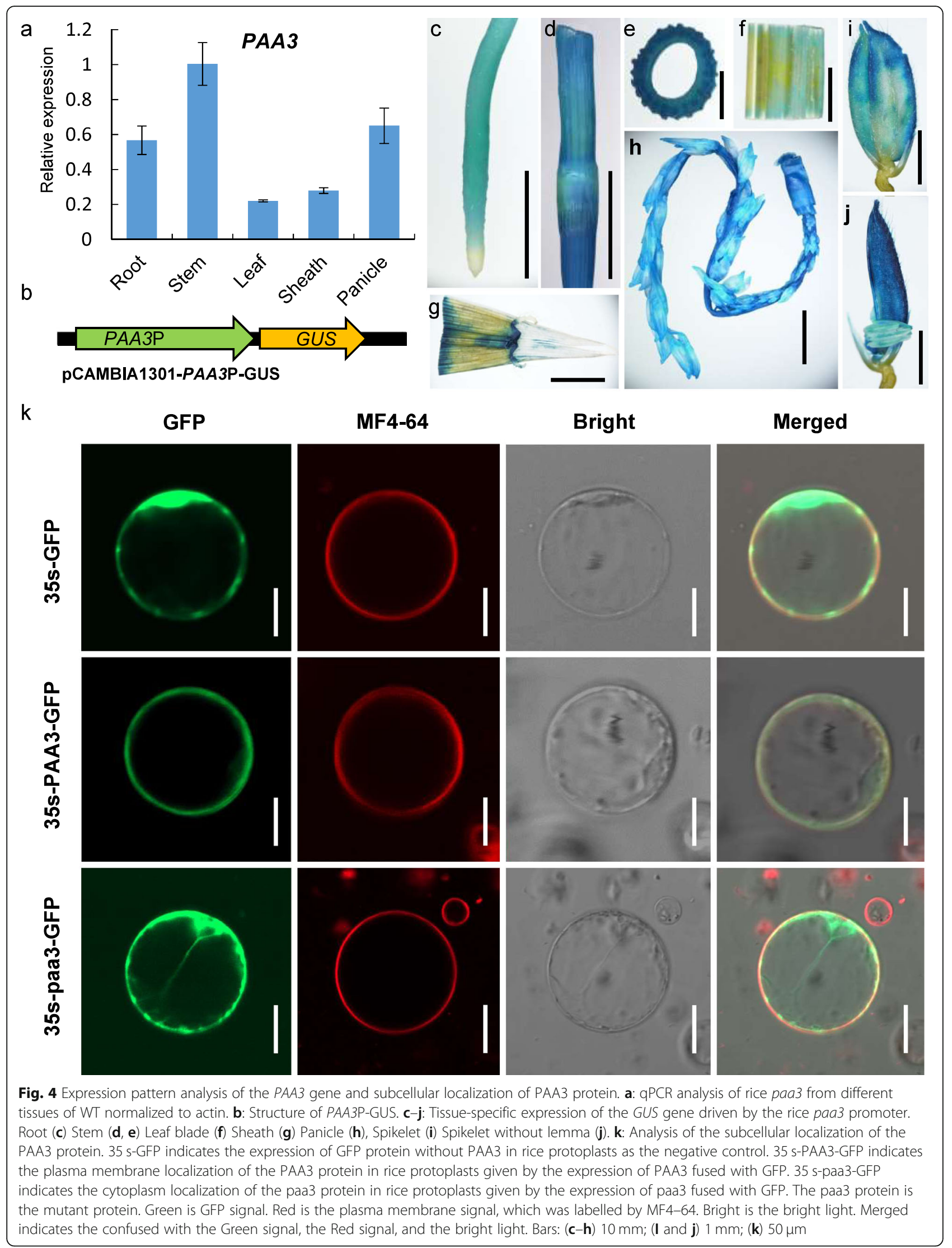




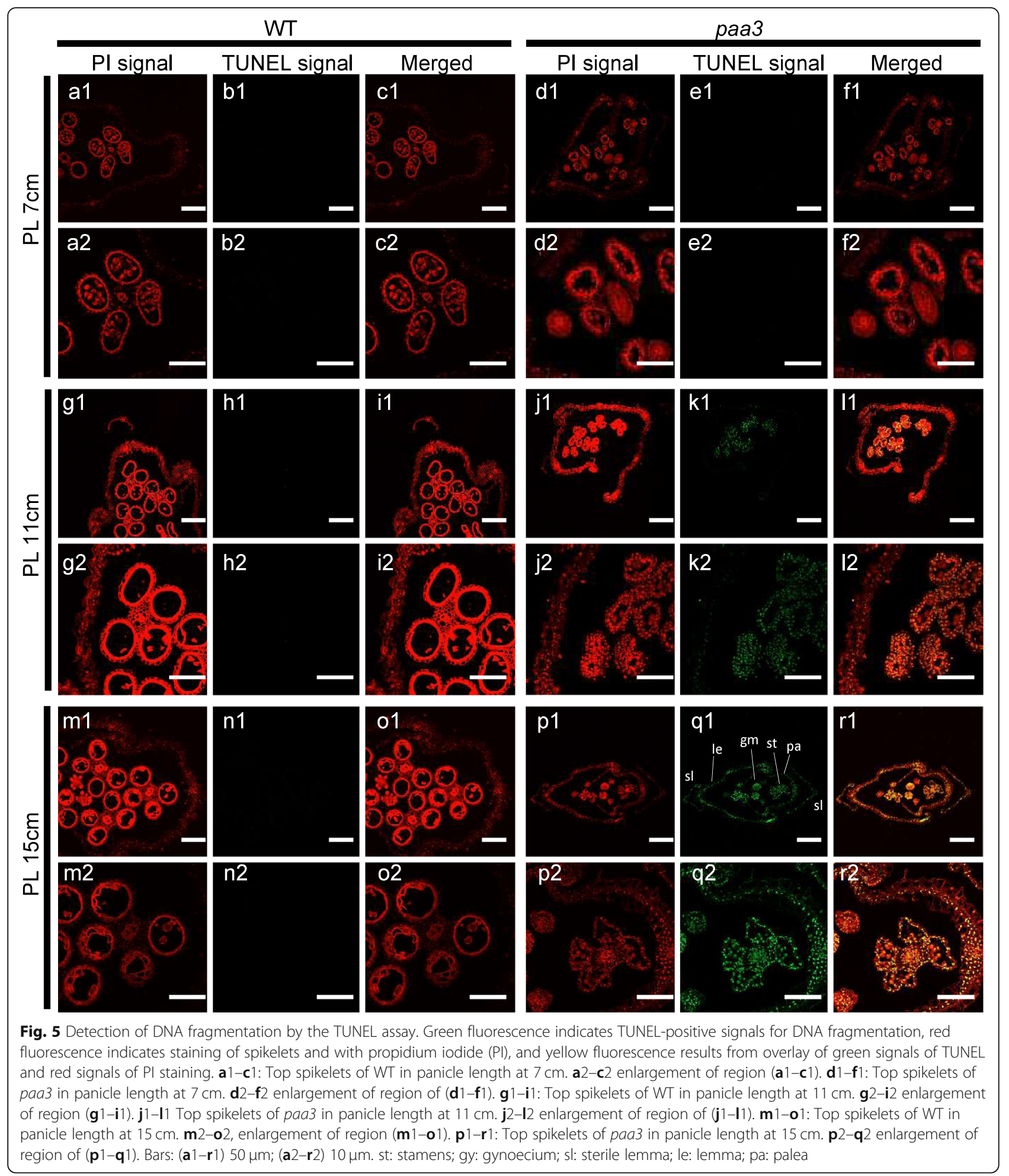

expression of OsVPE3 in the paa3 panicles was lower than that in the WT panicles at the $\sim 7 \mathrm{~cm}$ stage but was higher than that at the $\sim 11$ and $\sim 15 \mathrm{~cm}$ stages (Fig. 6d). Catalase (CAT) is the key peroxidase in the biological defensive system, playing a role in converting excessive $\mathrm{H}_{2} \mathrm{O}_{2}$ into oxygen. OsCATA, OsCATB, and OsCATC encode CAT isozymes in rice (Zhang et al., 2016). Our results show that all three genes 

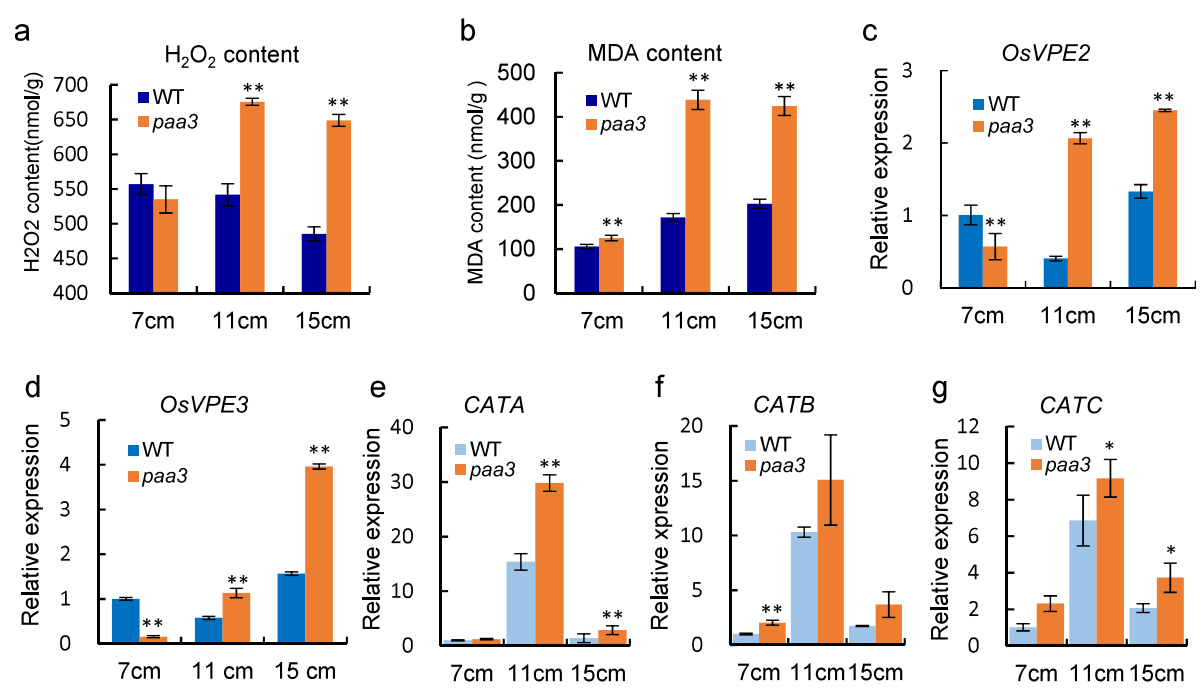

Fig. 6 Cell death-related Events are induced in paa3. a: Measurement of $\mathrm{H}_{2} \mathrm{O}_{2}$ content in panicles of the 7,11 , and $15 \mathrm{~cm}$ stages in WT and paa3. b: Measurement of MDA content in panicles of the 7, 11, and $15 \mathrm{~cm}$ stages in WT and paa3, showing overaccumulation of ROS during late development stages in paa3. c: Expression level of OsVPE2 in WT and paa3 panicles at the 7, 11, and $15 \mathrm{~cm}$ stages. d: Expression levels of OsVPE2 and OSVPE3 in WT and paa3 panicles at the 7, 11, and $15 \mathrm{~cm}$ stages, which showed PCD from the stage of $11 \mathrm{~cm}$. e-g: Relative expression of CATA, CATB, and CATC isozymes. (rice ACTIN was used as the internal control). * indicates $p<0.05$; ** indicates $p<0.01$ (Student's t-test)

were expressed at higher levels in paa3 than those in the WT panicles (Fig. 6e-g).

To provide further clarification of the mechanisms underlying ROS accumulation in the paa 3 panicle, we conducted transcriptome analysis. A total of 1075 differentially expressed genes (DEGs) were characterized, including 991 upregulated and 84 downregulated genes. The number of upregulated DEGs was 11.79 times the number of downregulated DEGs (Fig. S5). Next, Gene Ontology (GO) analysis showed that DEGs related to the oxidation response (GO: 0006979) and oxidoreductase activity (GO: 0016705) were enriched significantly, most of which were upregulated in the paa3 mutant (Fig. 7a, b). Further qPCR was used to verify the expression of some of these DEGs including LOC_Os04g10160, LOC_ Os01g43750, LOC_Os11g29290, and LOC_Os02g36030 in "oxidoreductase activity"; and LOC_OsO4g59190, LOC_ Os04g59150, LOC_Os01g73200, and LOC_Os04g59260 in response to "oxidative stress". Compared to the WT, expression of LOC_OsO4g10160 and LOC_Os04g59190 was upregulated nearly 20 -fold in the paa 3 mutant, and expression of the other genes was upregulated more than 4-fold (Fig. 7c). These results suggested that $P A A 3$ played an important role in removal of ROS in rice.

\section{Discussion}

In this study, we characterized a novel panicle development mutant paa3, which exhibited serious spikelet degeneration at the apical portion of panicle. Along with a reduced number of branches and 1000-grain weight, panicle apical portion resulted in a severe reduction of grain yield in the paa3 mutant. During rice growth and development, panicle development is crucial for grain yield in rice. Panicle apical portion is a common reason for the low seed-setting rate in rice (Heng et al., 2018). A number of environmental, physiological, and genetic factors can cause panicle apical portion and reduction in grain yield including climatic conditions (Yao et al., 2000), hormonal imbalance (Wang et al., 2018a, b), nutrient deficiency (Durbak et al., 2014), and mutations of genes including TUTOU1,OsALMT7, SPL6, OsCIPK31, and DPS1 (Bai et al., 2015; Heng et al., 2018; Peng et al., 2018; Wang et al., 2018a, b; Zafar et al., 2019). When panicle apical portion in tut1, spl6, and paa1019 mutants occurred before the heading stage, it occurred in late panicle development in the paab1-1 and dps1 mutants, similar to paa3 in this study. With the exception of the abortion phenotype, similar to tut 1 , the seed size of the paa3 also became smaller, while it did not change in the paab1-1, spl6, paa1019, and dps1 mutants. In addition, a series of agronomy traits, such as panicle length, number of spikelets per panicle, setting percentage, and 1000-grain weight were all decreased in these mutants. Therefore, these studies suggested that the genes related to panicle apical portion might have a wide effect on panicle development.

Rice grains are enclosed by the hull (lemma and palea), the development of which can largely determine the final size of rice grains and then affect the rice yield and quality (Li et al., 2018; Li et al., 2019). In recent years, many studies have shown that rice size are determined by numerous genes, most of which are involving in regulation 


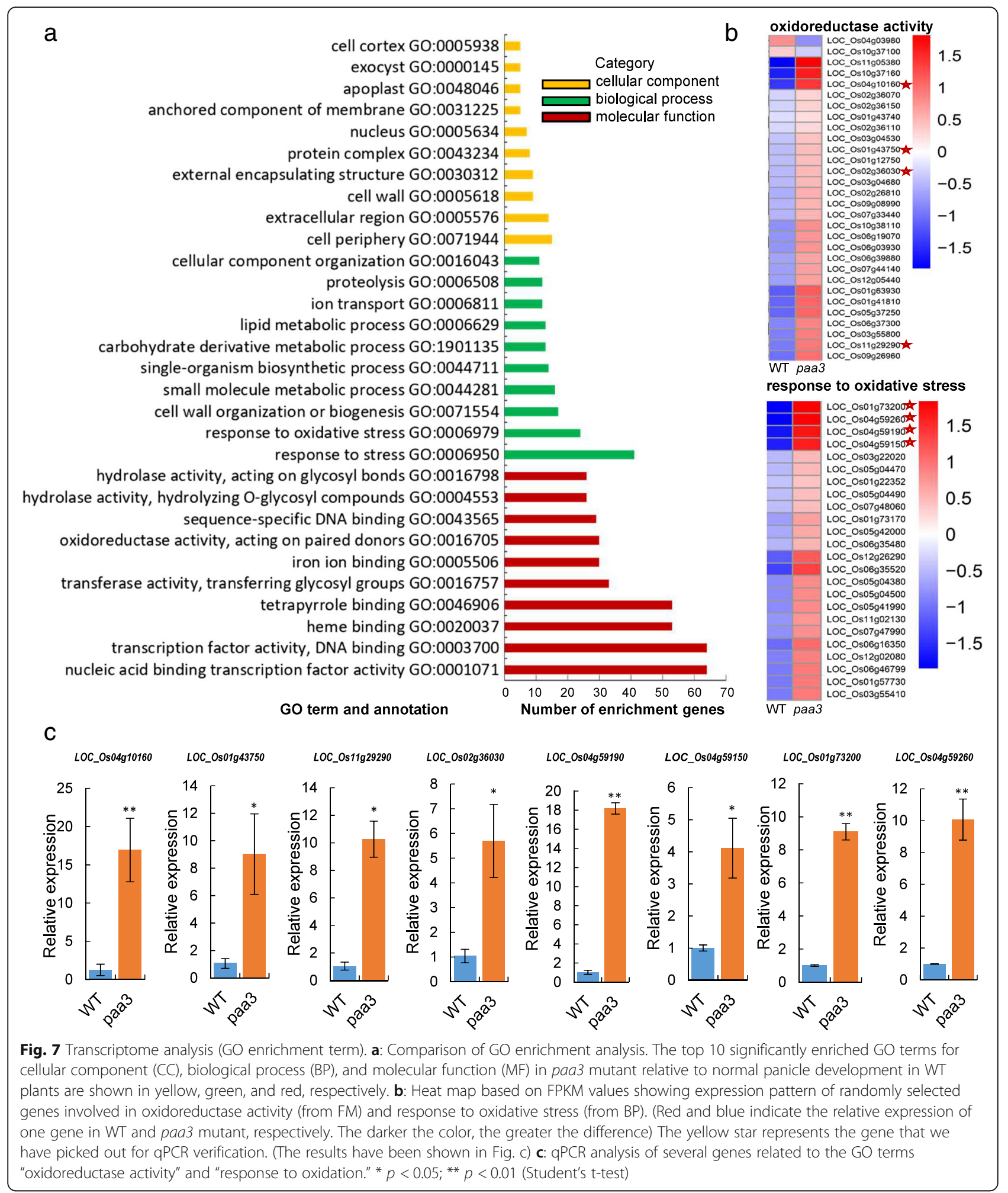

of hull development (cell differentiation and expansion) ( $\mathrm{Li}$ et al., 2018; Li et al., 2019; Fan \& Li, 2019). In our study, we observed the hull (lemma and palea) of paa3 mutant and WT with scanning electron microscopy, and it was obvious that the hull cells of the aborted spikelet in paa3 mutant were sharply smaller than those of WT (Fig. 2d). Then, we speculate that the hull cell extension of most of spikelets (including the fertile ones) in the paa3 mutant might be affected, causing the small size seeds. However, it was unclear whether the PAA3 gene 
regulated directly the hull development. It was possible that reduced size of grain might been indirect effect related to smaller panicle and reduced plant height in paa3 mutant.

PCD induced by ROS accumulation in the panicle might play a key role in most panicle abortion mutants in rice. OsALMT7 encoded an aluminum-activated malate transporter in rice. Malate was a central metabolite in the plant cell and was involved in the mitochondrial tricarboxylic acid and glyoxylate cycles in plant species, and can participate in redox reactions to produce NAD $(\mathrm{H})$ or NADP $(\mathrm{H})$ to maintain the balance of intracellular redox. In the paab1-1 mutant, the loss-of-function mutant of OsALMT7, reduced malate might disrupted the redox balance in the panicle cells, leading to the accumulation of ROS and the death of panicle cells (Heng et al., 2018). DPS1 encoded a CBSDUF protein, and could interact with $\operatorname{Trx}$ proteins (Trx1 and Trx20) to regulate ROS homeostasis in rice panicle development. Loss-of-function DPS1 gene accumulated more ROS in dps 1 mutant defective panicles, and also induced cell death and panicle apical degeneration (Zafar et al., 2019). In this study, our results also strongly supported the involvement of $P A A 3$ in ROS removal, and overaccumulation of ROS triggers PCD in the apical portion of the paa3 panicle.

PAA3 encoded OSA7, a plasma membrane $\mathrm{H}^{+}$-ATPase that was a member of the ATPase superfamily. The structure of the plasma membrane $\mathrm{H}^{+}$-ATPase was highly conserved from fungi to higher plants, with the exception of the C-terminal region (Wang et al., 2014). Depending on the structure of the C-terminal region, the plasma membrane $\mathrm{H}^{+}$-ATPase could be divided into two types: the penultimate threonine (Thr)-containing $\mathrm{H}^{+}$-ATPase (pT $\mathrm{H}^{+}$-ATPase) and the non-penultimate Thr-containing $\mathrm{H}^{+}$-ATPase (non-pT $\mathrm{H}^{+}$-ATPase) (Okumura et al., 2012); OSA7 belonged to the former group. A Tos17 insertion mutant of OSA7 resulted in impairment of the BL-induced stomatal opening, and ultimately a reduced transpiration rate, suggesting that OSA7 was involved in the Blue light-induced stomatal opening of dumbbell-shaped guard cells in monocotyledon species (Toda et al., 2016). The $\mathrm{H}^{+}$-ATPase is present in the membrane systems of fungi and plants widespread. In plants $\mathrm{H}^{+}$-ATPase releases protons from inside to outside the cell, and enhances membrane potential and $\mathrm{pH}$ gradient, contributing to cell wall acidification.The plasma membrane $\mathrm{H}^{+}$-ATPases were involved in many aspects of biology including BL-induced stomatal opening of dumbbell-shaped guard cells in the monocotyledon species, the uptake of phosphorus by the roots, and sustained pollen tube growth and fertilization (Chang et al., 2008; Toda et al., 2016; Hoffmann et al., 2020). There are $11 \mathrm{H}^{+}$-ATPase subtypes in Arabidopsis thaliana, including AHA1 which plays an important role in the blue-light dependent stomatal opening, AHA4 which plays a role in endodermal transport of solutes, and AHA10 which is associated with seed development (Yamauchi et al., 2016; Vitart et al., 2010; Appelhagen et al., 2015). Similarly, there are $10 \mathrm{H}^{+}$-ATPase in rice, including OSHA1 gene associated with the ability to absorb nutrients, OSHA2 may be related to photosensitive pigments and OSHA3 which can regulate spore development. (Wang et al., 2014; Luis et al., 2020; Zhang et al., 2019). However, to date only a few $\mathrm{H}^{+}$-ATPase isoforms had been identified and their physiological roles have proven difficult to analyze, given that no phenotypes of $\mathrm{H}^{+}$-ATPase mutant have been reported. In this study, the $P A A 3$ mutation resulted in excessive accumulation of ROS followed by PCD in the panicle, providing a novel perspective to explore the function of $\mathrm{H}^{+}$-ATPases in the future. The further study of $P A A 3$ gene was helpful to further understand the mechanism of panicle apical abortion at the cellular level.

\section{Conclusions}

A novel paa3 mutant was identified in rice and showed severe panicle apical apportion and semidwarf. The PAA3 encoded a $\mathrm{H}^{+}$-ATPase, which was a membrane protein and highly expressed in stems and panicle. The TUNEL assay showed that the DNA fragmentation and cell death in paa3 spikelets started to occur between the stages of $\sim 7$ and $\sim 11 \mathrm{~cm}$ panicles. DAB staining and measurement of $\mathrm{H}_{2} \mathrm{O}_{2}$ and MDA content showed overaccumulation of ROS in the paa 3 spikelets at the $\sim 11 \mathrm{~cm}$ stage. Staining with trypan blue showed cell death in paa3 spikelets at the stage of $\sim 13 \mathrm{~cm}$. The expression of genes involving in $\mathrm{H}_{2} \mathrm{O}_{2}$-induced PCD also indicated over-accumulation of ROS in the paa3 spikelets. Taken together, the results indicated that $P A A 3$ played an important role in maintaining the panicle development through ROS removal.

\section{Materials and Methods}

\section{Plant Materials and Growth Conditions}

In this study, the paa3 was derived from the ethyl methane sulfonate mutant library of the maintainer $X I D A 1 B(1 B)$. The paa3 mutant was crossed with the sterile line $56 \mathrm{~s}$ to obtain the $F_{1}$ generation, and the $F_{1}$ generation was self-crossed to obtain the F2 population. All plant materials were grown in the experimental fields of the Rice Research Institute of Southwest University (Chongqing, China). All $N$. benthamiana plants were grown in a greenhouse at the Rice Research Institute of Southwest University, Chongqing, China. 


\section{Agronomic Trait Analysis}

For agronomic trait measurement, rice plants were grown in experimental fields in Chongqing, China under natural conditions. Agronomic traits (comprising internodes length, plant height, panicle length, number of primary branch, number of secondary branch, number of spikelets per panicle, number of seeds per panicle, and 1000-grain weight) for each of the paa3 mutant and the wild type were analyzed at the mature stage with 10 replicates.

\section{Scanning Electron Microscopy}

The panicles in both the WT and the paa3 mutant were examined using a scanning electron microscope (SU3500; Hitachi, Tokyo, Japan) with a $-20^{\circ} \mathrm{C}$ cooling stage under a low-vacuum environment, when the paa3 panicle began to show a panicle abortion phenotype. At the flowering stage, spikelets from paa3 and WT plants were observed using a stereomicroscope (SMZ1500; Nikon, Tokyo, Japan).

\section{TUNEL Assay}

Apical spikelets of the WT and paa3 panicles at the panicle lengths 7,11 , and $15 \mathrm{~cm}$ were collected and fixed in formalin-acetone-alcohol solution for $48 \mathrm{~h}$, soaked in paraffin, embedded, sliced, and baked for 3 days. The spikelets were then dewaxed with xylene, dried, and incubated with protease $\mathrm{K}$ for $10 \mathrm{~min}$, soaked in phosphate-buffered saline (PBS) for $5 \mathrm{~min}$, and soaked in $4 \%$ methanol-free formaldehyde solution for $5 \mathrm{~min}$. After adding $50 \mu \mathrm{L} \mathrm{rTdT}$, the specimen was incubated at $37^{\circ} \mathrm{C}$ for $3 \mathrm{~h}$, followed by incubation with 2x SSC solution in the dark for $15 \mathrm{~min}$ and with PBS for $5 \mathrm{~min}$; this procedure was repeated three times. Specimens were incubated with propidium iodide (PI) solution for $15 \mathrm{~min}$ to prevent infiltration, and soaked in water for $5 \mathrm{~min}$; this procedure was repeated three times. Finally, the tablets were sealed with sealant (PBS and glycerin 1:1) for observation. The green fluorescence of fluorescein (TUNEL signal) and red fluorescence of propidium iodide were analysed at $488 \mathrm{~nm}$ (excitation) and $520 \mathrm{~nm}$ (detection), and $488 \mathrm{~nm}$ (excitation) and $610 \mathrm{~nm}$ (detection), respectively, under a confocal laser scanning microscope (LSM710; Zeiss, Jena, Germany).

\section{Staining and Quantitative Measurement of ROS and Measurement of MDA Content}

We monitored cellular ROS levels in apical degenerated spikelets using DAB staining to detect $\mathrm{H}_{2} \mathrm{O}_{2}$. According to a method described previously (Wu et al., 2017), we used DAB to stain the top spikelets of WT and paa3, and quantified ROS by measuring $\mathrm{H}_{2} \mathrm{O}_{2}$. Fresh panicles $(1 \mathrm{~g})$ were collected and then measured using the $\mathrm{H}_{2} \mathrm{O}_{2}$ assay kit (Nanjing Jiancheng Bioengineering Institute,
Nanjing, China). To measure MDA content, we collected $1 \mathrm{~g}$ spikelets from the apical part of the WT and paa3 panicles. Then, according to the instructions of the MDA Assay Kit (Nanjing Jiancheng Bioengineering Institute), we measured the MDA content. All experiments were conducted on panicles at the booting stage.

\section{Trypan Blue Staining}

Trypan blue staining was used to examine PCD. The panicles of mutant and WT at the $13 \mathrm{~cm}$ stage were immersed in a boiling solution of trypan blue for $10 \mathrm{~min}$, and then removed and left at room temperature for $12 \mathrm{~h}$. This was then immersed in a solution of chloral hydrate at a concentration of $2.5 \mathrm{mg} / \mathrm{mL}$ to allow decolourization. Finally, the decolourized samples were stored at $50 \%$ glycerin. Staining was observed using a stereomicroscope.

\section{Map-Based Cloning}

The paa3 mutant was crossed with the sterile line " $56 \mathrm{~S}$ " to generate $F_{1}$. The $58 \mathrm{~F}_{2}$ plants that exhibited a mutant phenotype were selected as the mapping population. SSR repeat markers from publicly available rice databases, including Gramene (http://www.gramene.org), the Rice Genomic Research Program (http://rgp.dna.affrc. go.jp/public data/caps/index.html), and in/del markers designed by our group according to re-sequence of $\mathrm{XD} 1 \mathrm{~B}$ and $56 \mathrm{~S}$ genome, were used for fine-mapping of $P A A 3$. The primer sequences used for mapping and identification of transgenic plants are listed in Table S2.

\section{Vector Construction and Transformation}

To construct the complementation plasmid, a $10,725 \mathrm{bp}$ genomic fragment that contained the $P A A 3$ coding sequence, coupled with the $3251 \mathrm{bp}$ upstream and $1068 \mathrm{bp}$ downstream sequences, was amplified using the primers $P A A 3$-com-F (EcoR1) and PAA3-com-R (HindIII). The fragment was inserted into the binary vector pCAMBIA1301 using the pEASY-Uni Seamless Cloning and Assembly Kit (TransGen, Beijing, China). The recombinant plasmids were transformed into the paa 3 mutant using the Agrobacterium tumefaciens-mediated transformation method as described previously (Zhuang et al., 2020). For PAA3::GUS assays, the promoter of $P A A 3$ gene (3138 bp) was amplified. The fragment was inserted into the binary vector PCAMBIA1301. Then the recombinant plasmids were transformed into the japonica cultivar ZHONGHUA 11 (ZH11) using the A. tumefaciensmediated transformation method as described previously (Zhuang et al., 2020). The primers used for vector construction are listed in Table S2. 


\section{PAA3P::GUS Staining}

For promoter activity analysis, a 3138 bp genomic fragment, which is the promoter of the PAA3 gene, was PCR-amplified from WT genomic DNA with the primer pair PAA3P-GUS-F and PAA3P-GUS-R (Table S2), and fused to the GUS reporter gene in the vector pCAMBIA1301. GUS staining was performed on PAA3P::GUS $\mathrm{T}_{0}$ generation transgenic plants in accordance with a previous method (Jefferson, 1989). After bleaching with ethanol, photographs were taken using a stereomicroscope.

\section{Subcellular Localization of the PAA3 (Transient Expression Assays in Rice Protoplasts)}

The full-length coding region (without the termination codon) of PAA3 was amplified using the SL-PAA3-F (Spe1) and SL-PAA3-R (Sma1) primers. The fragment was cloned into the expression cassette pAN580-35S:: GFP to generate the pAN580-35S::PAA3-GFP fusion vector. The pAN580-35S::GFP and pAN580-35S:: PAA3GFP plasmids were then transformed into rice protoplasts. After incubation for $12-16 \mathrm{~h}$ at $28^{\circ} \mathrm{C}$, GFP fluorescence was detected using a confocal laser scanning microscope (LSM710; Zeiss, Jena, Germany). Primers used for subcellular localization are listed in Table S2.

\section{Subcellular Localization of the PAA3(Transient Expression in Nicotiana benthamiana Leaves)}

The full-length coding region (without the termination codon) of PAA3 was amplified using the YC-PAA3-F (BamH1) and YC-PAA3-R (Xba1) primers. The fragment was cloned into the expression cassette pCAMBIA130035S::eGFP to generate the pCAMBIA-35S::PAA3-eGFP fusion vector. The pCAMBIA1300-35S::eGFP and pCAMBIA-35S::PAA3-eGFP plasmids were then transformed into Agrobacterium tumefaciens strain GV3101. And then Agrobacterium tumefaciens strain GV3101containing the target plasmid was grown in YEB medium with antibiotic selection to $\mathrm{OD}_{600}=0.6-0.8$. Cells were suspended to an $\mathrm{OD}_{600}=0.4$ in MES buffer $(10 \mathrm{mM}$ $\mathrm{MgCl}_{2}, 10 \mathrm{mM}$ MES; pH 5.6) and kept in the dark for 2$4 \mathrm{~h}$ before inoculation. Leaves were analyzed at $2-3$ days after transformation. Primers used for subcellular localization are listed in Table S2.

\section{DNA Extraction, RNA Isolation, and qPCR}

Total DNA from WT and paa3 mutant was extracted using the CTAB method. Total RNA from root, stem, leaf, sheath, panicle, bud, and shoot was isolated using the RNA prep Pure Plant Kit (Tiangen, Beijing, China). The first-strand complementary cDNA was synthesized from $2 \mu \mathrm{g}$ total RNA using oligo $(\mathrm{dT})_{18}$ primers in a $20 \mu \mathrm{L}$ reaction volume using the
PrimeScript ${ }^{\oplus}$ Reagent Kit with gDNA Eraser (Takara, Dalian, China). The qPCR analysis was performed using the $\mathrm{SYBR}^{\circ}$ Premix Ex $\mathrm{Taq}^{\mathrm{Ta}}$ II Kit (Takara) in the ABI 7500 Sequence Detection System (Applied Biosystems, Carlsbad, CA, USA). ACTIN (OsRac1, LOC_Os01g12900) was used as the endogenous control. At least three replicates were performed. Primers used for qPCR are listed in Table S2.

\section{RNA-Sequencing Analysis}

Analysis of RNA sequencing (RNA-seq) data was performed using a standard protocol (Trapnell et al., 2012). For RNA-seq analysis, RNA was extracted from WT and paa 3 panicles of $11 \mathrm{~cm}$ in length that corresponded to the developmental stage just after the start of panicle abortion. All sequencing samples were treated. RNA sequencing was performed by Novogene Biotechnology (Beijng, China), and sequencing data were retrieved through the standard Illumina pipeline with custom and default parameters. HTSeq software was used to analyse the original sequences of known genes for all the samples (Novogene Biotechnology, Beijing, China), and the expression of known genes was calculated using the fragments per kilobase of transcript per million fragments mapped (FPKM). HTSeq was used to estimate gene expression levels. DEGs were identified considered $P \leq 0.05$ and a $\log 2$ fold-change $\geq 1$. Clusters were analysed by principal component analysis, and DEGs were analysed by DESeq, with a cut-off $\mathrm{P} \leq 0.05$ and fold change $\geq 2$. GO analysis and Kyoto Encyclopedia of Genes and Genomes analyses were performed to identify the significantly enriched biological processes in paa3.

\section{Abbreviations}

PAA3: PANICLE APICAL ABORTION 3; WT: Wild-type; paa3: Panicle apical abortion 3; AMs: Axillary meristems; PB: Primary branches; SB: Secondary branches; LS: Lateral spikelet; TS: Terminal spikelet; QTL: Quantitative trait locus; ROS: Reactive oxygen species; PCD: Programmed cell death;

TUNEL: Terminal deoxynucleotidyl transferase-mediated dUTP nick-end labeling; BSA: Bulked segregant analysis; MDA: Malondialdehyde; SSR: Simple sequence repeat; DAB: 3,3'-Diaminobenzidine; EMS: Ethyl methane sulfonate; CTAB: cetyltrimethylammonium bromide; GO: Gene ontology; KEGG: Kyoto encyclopedia of genes and genomes; GFP: Green fluorescent protein;

GUS: $\beta$ - Glucuronidase; qPCR: Quantitative PCR; TEM: Transmission electron microscopy

\section{Supplementary Information}

The online version contains supplementary material available at https://doi. org/10.1186/s12284-021-00509-5.

Additional file 1: Fig. S1. Identification of transgenic plants. a: Two pairs of primers for amplification to detect the 15 transformants (comF1GUSR1 for exogenous vector; comF2-comR2 for endogenic sites). b, Comparison diagram of sequence.

Additional file 2: Fig. S2. Transient expression in Nicotiana benthamiana leaves. Upper row indicates the expression of GFP protein without PAA3 in $\mathrm{N}$ benthamiana leaves as the negative control. Lower row indicates the plasma membrane localization of the PAA3 protein in $\mathrm{N}$ benthamiana leaves given by the expression of PAA3 fused with GFP. 
Green is GFP signal. The plasma membrane as labelled by MF4-64. Red is the chloroplast signal. Bars: $20 \mu \mathrm{m}$.

Additional file 3: Fig. S3. Trypan blue staining analysis. a, Top panicle of paa3 mutants and WT. (panicle length $=13 \mathrm{~cm}$ ). b, Top spikelet of paa3 mutants and WT. Bars: (a, b) $5 \mathrm{~mm}$.

Additional file 4: Fig. S4. DAB staining analysis. a, Top panicle of paa3 mutants and WT. (panicle length $=13 \mathrm{~cm}$ ). b, Top spikelet of paa3 mutants and WT. Bars: (a, b) $5 \mathrm{~mm}$.

Additional file 5: Fig. S5. Transcriptome analysis of paa3 mutant. a: It showed that there were 1075 differential genes in WT and paa3 samples, among which 991 genes were up-regulated and 84 genes were downregulated. b: The volcano figure showed an overall overview, including 28,317 genes with no change in expression, 991 up-regulated genes and 84 down-regulated genes.

Additional file 6: Table S1. Total frequency of spikelet abortion of paa3 mutant.

Additional file 7: Table S2. All primers used for this study.

\section{Acknowledgements}

We thank Professor Ming Luo for helping in subcellular localization.

\section{Authors' Contributions}

YFL and FYY designed the research; FYY and MJH performed the mappingbased clone and phenotype analyses; FYY and ZCL performed the TUNEL assay; FYY, HZ, and RC performed the experiments to determine the subcellular localization of PAA3 protein; FYY and MX performed the GPCR; JT, YW, QLC, ZYW, HHZ and LL contributed to the data analysis; FYY, YFL and MX wrote the manuscript; All authors read and approved the final manuscript.

\section{Funding}

This work was supported by the National Natural Science Foundation of China (No. 31971919), the Natural Science Foundation Project of Chongqing Science and Technology Commission (No. cstc2020jcyj-jqX0020), and Chongqing Graduate Research and Innovation Project funding (No. CYS20123).

\section{Availability of Data and Materials}

The datasets supporting the conclusions of this article are included within the article and its additional files.

\section{Declarations}

Ethics Approval and Consent to Participate

Not applicable.

\section{Consent for Publication}

Not applicable.

\section{Competing Interests}

The authors declare that they have no competing interests.

Received: 4 February 2021 Accepted: 9 June 2021

Published online: 15 July 2021

\section{References}

Appelhagen I, Nordholt N, Seidel T, Spelt K, Koes R, Quattrochio F, Sagasser M, Weisshaar B (2015) TRANSPARENT TESTA 13 is a tonoplast P3A -ATPase required for vacuolar deposition of proanthocyanidins in Arabidopsis thaliana seeds. Plant J 82(5):840-849. https://doi.org/10.1111/tpj.12854

Ashikari M, Sakakibara H, Lin S, Yamamoto T, Takashi T, Nishimura A, Angeles ER Qian Q, Kitano H, Matsuoka M (2005) Cytokinin oxidase regulates Rice grain production. Science 309(5735):741-745. https://doi.org/10.1126/science.11133 73

Bai J, Zhu X, Wang Q, Zhang J, Chen H, Dong G, Zhu L, Zheng H, Xie Q, Nian J, Chen F, Fu Y, Qian Q, Zuo J (2015) Rice TUTOU1 encodes a suppressor of CAMP receptor-like protein that is important for actin Organization and panicle development. Plant Physiol 169(2):1179-1191. https://doi.org/10.11 04/pp.15.00229
Chang C, Hu Y, Sun S, Zhu Y, Ma G (2008) Xu G (2008) proton pump OsA8 is linked to phosphorus uptake and translocation in rice. J Exp Bot 60(2):557565. https://doi.org/10.1093/jxb/ern298

Chen THH, Murata N (2002) Enhancement of tolerance of abiotic stress by metabolic engineering of betaines and other compatible solutes. Curr Opin Plant Biol 5(3):250-257. https://doi.org/10.1016/S1369-5266(02)00255-8

Deng M, Bian H, Xie Y, Kim Y, Wang W, Lin E, Zeng Z, Guo F, Pan J, Han N, Wang J, Qian Q, Zhu M (2011) Bcl-2 suppresses hydrogen peroxide-induced programmed cell death via OSVPE2 and OSVPE3, but not via OsVPE1 and OsVPE4, in rice. FEBS J 278(24):4797-4810. https://doi.org/10.1111/j.1742-4 658.2011.08380.x

Durbak AR, Phillips KA, Pike S, O'Neill MA, Mares J, Gallavotti A, Malcomber ST, Gassmann W, McSteen P (2014) Transport of boron by the tassel-less1 aquaporin is critical for vegetative and reproductive development in maize. Plant Cell 26(7):2978-2995. https://doi.org/10.1105/tpc.114.125898

Fan YW, Li YB (2019) Molecular, cellular and yin-yang regulation of grain size and number in rice. Mol Breed 39(12):1-25

Heng Y, Wu C, Long Y, Luo S, Ma J, Chen J, Liu J, Zhang H, Ren Y, Wang M, Tan J, Zhu S, Wang J, Lei C, Zhang X, Guo X, Wang H, Cheng Z, Wan J (2018) OSALMT7 maintains panicle size and grain yield in Rice by mediating malate transport. Plant Cell 30(4):889-906. https://doi.org/10.1105/tpc.17.00998

Hoffmann RD, Portes MT, Olsen LI, Damineli DSC, Hayashi M, Nunes CO, Pedersen JT, Lima PT, Campos C, Feijo JA, Palmgren M (2020) Plasma membrane H(+ )-ATPases sustain pollen tube growth and fertilization. Nat Commun 11(1): 2395

Huang X, Qian Q, Liu Z, Sun H, He S, Luo D, Xia G, Chu C, Li J, Fu X (2009) Natural variation at the DEP1 locus enhances grain yield in rice. Nat Genet 41(4):494-497. https://doi.org/10.1038/ng.352

Huijser P, Schmid M (2011) The control of developmental phase transitions in plants. Development 138(19):4117-4129. https://doi.org/10.1242/dev.063511

Ikeda-Kawakatsu K, Maekawa M, Izawa T, Itoh Jl, Nagato Y (2012) ABERRANT PANI CLE ORGANIZATION 2/RFL, the rice ortholog of Arabidopsis LEAFY, suppresses the transition from inflorescence meristem to floral meristem through interaction with APO1. Plant J 69(1):168-180. https://doi.org/1 0.1111/j.1365-313X.2011.04781.X

Kato Y, Hirotsu S, Nemoto K, Yamagishi J (2008) Identification of QTLs controlling rice drought tolerance at seedling stage in hydroponic culture. Euphytica 160(3):423-430. https://doi.org/10.1007/s10681-007-9605-1

Kobayasi K, Yamane K, Imaki T (2001) Effects of non-structural carbohydrates on spikelet differentiation in Rice. Plant Prod Sci 4(1):9-14. https://doi.org/10.162 6/pps.4.9

Komatsu K, Maekawa M, Ujiie S, Satake Y, Furutani I, Okamoto H, Shimamoto K, Kyozuka J (2003) LAX and SPA: major regulators of shoot branching in Rice. Proc Natl Acad Sci U S A100(20):11765-11770

Kuroyanagi M, Yamada K, Hatsugai N, Kondo M, Nishimura M, Hara-Nishimura I (2005) Vacuolar processing enzyme is essential for mycotoxin-induced cell death in Arabidopsis thaliana. J Biol Chem 280(38):32914-32920. https://doi. org/10.1074/jbc.M504476200

Li M, Tang D, Wang KJ, Wu XR, Lu LL, Yu HX, Gu MH, Yan CJ, Cheng ZK (2011) Mutations in the F-box gene LARGER PANICLE improve the panicle architecture and enhance the grain yield in rice. Plant Biotechnol. J 9(9): 1002-1013

Li N, Xu R, Duan PG, Li YH (2018) Control of grain size in rice. Plant Reprod 31(3): 237-251. https://doi.org/10.1007/s00497-018-0333-6

Li N, Xu R, Li YH (2019) Molecular networks of seed size control in plants. Annu Rev Plant Biol, 70(undefined), 435-463. doi:https://doi.org/10.1146/annurev-a rplant-050718-095851, 1

Li S, Zhao B, Yuan D, Duan M, Qian Q, Tang L, Wang B, Liu X, Zhang J, Wang J, Sun J, Liu Z, Feng Y-Q, Yuan L, Li C (2013) Rice zinc finger protein DST enhances grain production through controlling Gn1a/OsCKX2 expression. Proc Natl Acad Sci 110(8):3167-3172. https://doi.org/10.1073/pnas. 1300359110

Li X, Qian Q, Fu Z, Wang Y, Xiong G, Zeng D, Wang X, Liu X, Teng S, Hiroshi F, Yuan M, Luo D, Han B, Li JY (2003) Control of tillering in rice. Nature 422(6932):618-621. https://doi.org/10.1038/nature01518

Lu Z, Yu H, Xiong G, Wang J, Jiao Y, Liu G, Jing Y, Meng X, Hu X, Qian Q, Fu X, Wang Y, Li JY (2013) Genome-wide binding analysis of the transcription activator IDEAL PLANT ARCHITECTURE1 reveals a complex network regulating Rice Plant ARCHITECTURE. Plant Cell 25(10):3743-3759. https://doi. org/10.1105/tpc.113.113639

Luis RVJ, Carles B, Manuel T, Concha D (2020) The G123 rice mutant, carrying a mutation in SE13, presents alterations in the expression 
patterns of photosynthetic and major flowering regulatory genes. PLoS One 15(5)

Mittler R (2017) ROS are good. Trends Plant Sci 22(1):11-19. https://doi.org/10.101 6/j.tplants.2016.08.002

Nakagawa M, Shimamoto K, Kyozuka J (2002) Overexpression of RCN1 and RCN2, rice TERMINAL FLOWER 1/CENTRORADIALIS homologs, confers delay of phase transition and altered panicle morphology in rice. Plant J 29(6):743750. https://doi.org/10.1046/j.1365-313X.2002.01255.x

Okumura M, Inoue SI, Takahashi K, Ishizaki K, Kohchi T, Kinoshita T (2012) Characterization of the plasma membrane $\mathrm{H}^{+}$-ATPase in the liverwort Marchantia polymorpha. Plant Physiol 159(2):826-834. https://doi.org/10.11 04/pp.112.195537

Peng YB, Hou FX, Bai Q, Xu PZ, Liao YX, Zhang HY, Gu CJ, Deng XS, Wu TK, Chen $X Q$, Ali A, Wu X (2018) Rice Calcineurin B-like protein-interacting protein kinase 31 (OsCIPK31) is involved in the development of panicle apical Spikelets. Front Plant Sci 9:1661. https://doi.org/10.3389/fpls.2018.01661

Rao NN, Prasad K, Kumar PR, Vijayraghavan U (2008) Distinct regulatory role for $\mathrm{RFL}$, the rice LFY homolog, in determining flowering time and plant architecture. Proc Natl Acad Sci U S A 105(9):3646-3651. https://doi.org/10.1 073/pnas.0709059105

Sakamoto T, Matsuoka M (2004) Generating high-yielding varieties by genetic manipulation of plant architecture. Curr Opin Biotechnol 15(2):144-147. https://doi.org/10.1016/j.copbio.2004.02.003

Tabuchi H, Zhang Y, Hattori S, Omae M, Shimizu-Sato S, Oikawa T, Qian Q, Nishimura M, Kitano H, Xie H, Fang X, Yoshida H, Kyozuka J, Chen F, Sato Y (2011) LAX PANICLE2 of Rice encodes a novel nuclear protein and regulates the formation of axillary meristems. Plant Cell 23(9):3276-3287. https://doi. org/10.1105/tpc. 111.088765

Takeda S, Matsuoka M (2008) Genetic approaches to crop improvement: responding to environmental and population changes. Nat Rev Genet 9(6): 444-457. https://doi.org/10.1038/nrg2342

Toda Y, Wang Y, Takahashi A, Kawai Y, Tada Y, Yamaji N, Feng Ma J, Ashikari M, Kinoshita T (2016) Oryza sativaH+-ATPase (OSA) is involved in the regulation of dumbbell-shaped guard cells of Rice. Plant Cell Physiol 57(6):1220-1230. https://doi.org/10.1093/pcp/pcw070

Trapnell C, Roberts A, Goff L, Pertea G, Kim D, Kelley DR, Pimentel H, Salzberg SL, Rinn JL, Pachter L (2012) Differential gene and transcript expression analysis of RNA-seq experiments with TopHat and cufflinks. Nat Protoc 7(3):562-578 https://doi.org/10.1038/nprot.2012.016

Vitart V, Baxter I, Doerner P, Harper JF (2010) Evidence for a role in growth and salt resistance of a plasma membrane $\mathrm{H}^{+}$-ATPase in the root endodermis. Plant J 27(3):191-201

Wang Y, Shimazaki K, Kinoshita T (2014) Multiple roles of the plasma membrane H(+)-ATPase and its regulation. Enzymes 35:191. https://doi.org/10.1016/ B978-0-12-801922-1.00008-7

Wang QL, Sun AZ, Chen ST, Chen LS, Guo FQ (2018a) SPL6 represses signalling outputs of ER stress in control of panicle cell death in rice. Nat Plants 4(5): 280-288. https://doi.org/10.1038/s41477-018-0131-z

Wang ZQ, Zhang WY, Yang JC (2018b) Physiological mechanism underlying spikelet degeneration in rice. J Integr Agric 17(7):1475-1481. https://doi.org/1 0.1016/S2095-3119(18)61981-1

Wu JG, Yang RX, Yang ZR, Yao SG, Zhao SS, Wang Y, Li PC, Song XW, Jin L, Zhou T, Lan Y, Xie LH, Zhou XP, Chu CC, Qi YJ, Cao XF, Li Y (2017) ROS accumulation and antiviral defence control by microRNA528 in rice. Nat Plants 3(1):16203. https://doi.org/10.1038/nplants.2016.203

Xing YZ, Zhang QF (2010) Genetic and molecular bases of Rice yield. Annu Rev Plant Biol 61(1):421-442. https://doi.org/10.1146/annurev-arplant-042809-1122 09

Yamauchi S, Takemiya A, Sakamoto T, Kurata T, Tsutsumi T, Kinoshita T (2016) The plasma membrane $\mathrm{H}^{+}$-ATPase AHA1 plays a major role in stomatal opening in response to blue light. Plant Physiol 171(4):2731-2743. https://doi.org/1 $0.1104 /$ pp. 16.01581

Yang J, Cho LH, Yoon J, Yoon H, Wai AH, Hong WJ, Han M, Sakakibara H, Liang W, Jung KH, Jeon JS, Koh HJ, Zhang DB, An G (2019) Chromatin interacting factor OsVIL2 increases biomass and rice grain yield. Plant Biotechnol J 17(1): 178-187

Yao Y, Yamamoto Y, Yoshida T, Nitta Y, Miyazaki A (2000) Response of differentiated and degenerated spikelets to top-dressing, shading and day/ night temperature treatments in rice cultivars with large panicles. Soil Sci Plant Nutr 46(3):631-641
Yoshida A, Sasao M, Yasuno N, Takagi K, Daimon Y, Chen RH, Yamazaki R, Tokunaga H, Kitaguchi Y, Sato Y, Nagamura Y, Ushijima T, Kumamaru T, lida S, Maekawa M, Kyozuka J (2013) TAWAWA1, a regulator of rice inflorescence architecture, functions through the suppression of meristem phase transition. Proc Natl Acad Sci U S A 110(2):767-772. https://doi.org/10.1073/pnas.12161 51110

Zafar SA, Patil SB, Uzair M, Fang J, Zhao J, Guo T, Yuan S, Uzair M, Luo Q, Shi J, Schreiber L, Li XY (2019) DEGENERATED PANICLE AND PARTIAL STERILITY 1 (DPS 1) encodes a cystathionine $\beta$-synthase domain containing protein required for anther cuticle and panicle development in rice. New Phytol 225: $356-375$

Zhang F, Zhang YC, Liao JY, Yu Y, Zhou YF, Feng YZ, Yang YW, Lei MQ, Bai M, Wu H, Chen YQ (2019) The subunit of RNA N6-methyladenosine methyltransferase OsFIP regulates early degeneration of microspores in rice. PLoS Genet 15(5):e1008120. https://doi.org/10.1371/journal.pgen.1008120

Zhang ZS, Xu YY, Xie ZW, Li XY, He ZH, Peng XX (2016) Association-dissociation of Glycolate oxidase with catalase in Rice: a potential switch to modulate intracellular $\mathrm{H}_{2} \mathrm{O}_{2}$ levels. Mol Plant 9(5):737-748. https://doi.org/10.1016/j. molp.2016.02.002

Zhuang H, Wang HL, Zhang T, Zeng XQ, Chen H, Wang ZW, Zhang J, Zheng H, Tang J, Ling YH, Yang ZL, He GH, Li YF (2020) NONSTOP GLUMES1 encodes a $\mathrm{C} 2 \mathrm{H} 2$ zinc finger protein that regulates spikelet development in rice. Plant Cell 32(2):392-413. https://doi.org/10.1105/tpc.19.00682

\section{Publisher's Note}

Springer Nature remains neutral with regard to jurisdictional claims in published maps and institutional affiliations.

\section{Submit your manuscript to a SpringerOpen ${ }^{\circ}$ journal and benefit from:}

- Convenient online submission

- Rigorous peer review

- Open access: articles freely available online

- High visibility within the field

- Retaining the copyright to your article

Submit your next manuscript at $\boldsymbol{\nabla}$ springeropen.com 